\title{
Physics of the Applegate mechanism: Eclipsing time variations from magnetic activity
}

\author{
M. Völschow ${ }^{1}$, D. R. G. Schleicher ${ }^{2}$, R. Banerjee ${ }^{1}$, and J. H. M. M. Schmitt ${ }^{1}$ \\ 1 Hamburger Sternwarte, Universität Hamburg, Gojenbergsweg 112, 21029 Hamburg, Germany \\ e-mail: marcel . voelschow@hs . uni-hamburg.de \\ 2 Departamento de Astronomía, Facultad Ciencias Físicas y Matemáticas, Universidad de Concepción, Av. Esteban Iturra s/n Barrio \\ Universitario, Casilla 160-C, Concepción, Chile
}

Received 26 May 2018 / Accepted 4 September 2018

\begin{abstract}
Since its proposal in 1992, the Applegate mechanism has been discussed as a potential intrinsical mechanism to explain transit-timing variations in various types of close binary systems. Most analytical arguments presented so far focused on the energetic feasibility of the mechanism while applying rather crude one- or two-zone prescriptions to describe the exchange of angular momentum within the star. In this paper, we present the most detailed approach to date to describe the physics giving rise to the modulation period from kinetic and magnetic fluctuations. Assuming moderate levels of stellar parameter fluctuations, we find that the resulting binary period variations are one or two orders of magnitude lower than the observed values in RS-CVn like systems, supporting the conclusion of existing theoretical work that the Applegate mechanism may not suffice to produce the observed variations in these systems. The most promising Applegate candidates are low-mass post-common-envelope binaries with binary separations $\lesssim 1 R_{\odot}$ and secondary masses in the range of $0.30 M_{\odot}$ and $0.36 M_{\odot}$.
\end{abstract}

Key words. stars: activity - stars: interiors - stars: AGB and post-AGB - binaries: eclipsing

\section{Introduction}

Precise timing measurements in close binaries routinely reveal variations in the eclipse timings. While for binaries with very short periods of less than three hours gravitational wave emission is the dominating means of angular momentum loss, magnetic braking can account for such effects in binaries with longer periods (see, e.g., Kraft et al. 1962; Faulkner 1971; Verbunt \& Zwaan 1981; Parsons et al. 2013). A subclass of close binaries, mainly RS Canum Venaticorum (RS CVn) and postcommon envelope binary (PCEB) systems, feature cyclic or nearly-periodic orbital period variations on timescales of a few years to decades that are incompatible with classical gravitational wave or magnetic braking models (see, e.g., Lanza et al 1998; Brinkworth et al. 2006; Zorotovic \& Schreiber 2013). For some systems, planetary companions may explain these variations (see, e.g., Qian et al. 2011; Beuermann et al. 2013; Nasiroglu et al. 2017; Han et al. 2017), while some of the planetary solutions were found to be dynamically unstable (Horner et al. 2013) or have been proven incorrect observationally (Hardy et al. 2015). On the other hand, the period variations in other systems such as QS Vir are still not well understood (Parsons et al. 2010).

Based on and inspired by earlier work by Matese \& Whitmire (1983) and Applegate \& Patterson (1987), Applegate (1992) proposed a new mechanism to explain cyclic orbital period variations in close binaries. The author assumed a time-dependent gravitational quadrupole moment modulated by the stellar activity cycle. Given a constant orbital angular momentum, an increasing quadrupole moment results in a stronger gravitational field and finally in a decreasing orbital radius and increasing orbital velocity, which can be observed as a reduction of the binary period.

To calculate the required energy as well as the expected quadrupole changes, Applegate (1992) considered a thin shell rotating in a point mass potential representing the rest of the star. The author assumed that the torque necessary to perform the angular momentum exchange is provided by subsurface magnetic fields of a few kG. Given such a field, luminosity variations on the $10 \%$ level and angular velocity variations on the $1 \%$ level are expected, leading to binary period variations compatible with the observed values in RS CVn systems $\left(\Delta P / P \sim 10^{-5}\right)$. Applegate (1992) made a number of testable predictions, which included significant period luminosity changes due to temperature variations and a 1:1 relation between the modulation period and the stellar activity cycle. Both processes have been observed in systems such as CG Cygni, accompanied by orbital period variations (Hall 1991), while luminosity variations can be observed by studying photospheric temperatures (see, e.g., Gray \& Baliunas 1994).

Lanza et al. (1998) generalized the magnetohydrodynamic aspects of the pioneering work of Applegate (1992), included the effects of internal magnetic fields, and elaborated on the connection between the Applegate mechanism and different types of dynamo models, emphasizing that a careful study of the Applegate mechanism may allow distinguishing between them. The improved Lanza et al. (1998) model is based on the momentum balance equation and assumes a uniform ratio of plasma pressure to magnetic pressure over the star. Expansion of the gravitational potential in spherical harmonics allowed Lanza et al. (1998) to calculate the quadrupole potential at the surface of the star, finding that the angular velocity changes required to produce a 
certain amount of period variation are reduced by a factor of 2 compared to the original Applegate (1992) approach.

Furthermore, Lanza et al. (1998) found that the driver of the period variations is the magnetic dynamo of the active component, which can effectively transform kinetic energy from nuclear reactions into magnetic energy and is more likely to be an $\alpha^{2} \Omega$ dynamo, introducing a further observational aspect. Lanza \& Rodonò (1999) stressed that the original Applegate (1992) ansatz may systematically overestimate the energies required to power the quadrupole moment variations as the exchanges between kinetic and magnetic energy are cyclic and partially reversible processes. Therefore, the authors concluded that the original model only provides upper limits for the required energy.

Lanza (2005) revisited the original work by Applegate (1992) and introduced an entirely new approach. Assuming that the angular velocity of the active component is only a function of the distance from its rotation axis, Lanza (2005) considered angular momentum redistributions not only between two layers, but rather general radial transport and redistribution modes within the convection zone. Imposing a strictly adiabatic convection zone and neglecting density perturbations, the author considered the equations of mass continuity and angular momentum conservation within a mean-field framework. Solving the angular momentum equation, Lanza (2005) calculated individual angular momentum redistribution modes from which he derived both the amplitude of the quadrupole moment change and the kinetic energy dissipated during the redistribution processes. Contrary to prior works, Lanza (2005) found that the power required to drive the observed levels of binary period variation in HR 1099 exceeds the luminosity provided by the active component by one or two orders of magnitude. Lanza (2006) extended the Lanza (2005) approach to angular velocity distributions that are functions of both radius and latitude, finding that the Applegate effect is still not a viable option in the case of HR 1099 and RS CVn systems in general.

Another route has been taken by Brinkworth et al. (2006), who generalized the original Applegate (1992) thin-shell ansatz to angular momentum exchanges between a finite core and a finite shell, including the core back-reaction to oblateness changes of the surrounding shell. Völschow et al. (2016) extended the Brinkworth et al. (2006) model to realistic stellar density profiles, derived analytic expressions to estimate the energy required to produce a certain level of period variation in a given system, and applied their full model to a set of 16 close binary systems, including 11 PCEBs. In line with previous works, the authors found that the Applegate effect cannot uniquely explain period variations in close binaries, and only 4 of the 16 systems were identified as potential Applegate candidates. Most recently, Navarrete et al. (2018) employed the analytic two-zone model by Völschow et al. (2016) to investigate how rotation affects the energetic feasibility of the Applegate effect and on which scale the activity cycle matches the observed modulation period, noting that the systems with the highest rotation rates are the most likely Applegate candidates. However, one critical simplification of both the Brinkworth et al. (2006) and Völschow et al. (2016) models is that the authors set the core-shell transition where it minimizes the energy necessary to power the Applegate effect.

In this paper, we extend the Lanza (2006) model by assuming a time-dependent magnetic field, velocity field fluctuations, and magnetic field fluctuations in the convection zone from which we can explicitly calculate the temporal evolution of the dissipated energy as well as the binary period variation. In addition, we consider superpositions of angular momentum redistribution modes instead of calculating the quadrupole moment changes caused by individual modes, as previously done by Lanza (2005, 2006). For the sake of consistency, we first apply our model to HR 1099 and use the system as an illustrative example for the basic predictions we make, before we extend our analysis to PCEB systems and identify the most likely Applegate mechanism candidates.

\section{Model description}

The formalism we employed is based on Lanza (2006). Sections 2.1 and 2.2.1 closely follow the author's description, but our presentation puts an emphasis on a more algorithmic description. Starting from Sect. 2.3.1, we extend on the crucial aspects of the calculation by making an ansatz for the stellar parameter fluctuations, explicitly solving the temporal part of the underlying differential equation (see Sect. 2.2.1), elaborating on the evolution of the mechanism, and presenting an approach for describing superpositions of angular momentum redistribution modes.

\subsection{Setup}

Lanza (2006) assumed an inertial reference frame originating at the barycenter of the active component star, with the $z$-axis pointing in the direction of the stellar rotation axis. In line with Lanza (2006), we employ a spherical coordinate system where $r$ is the distance from the origin, the colatitude $\theta$ is measured from the north pole and the azimuthal angle is $\phi$, and we impose that all variables are independent of $\phi$. The hydrodynamics of the turbulent convection zone are described employing a meanfield approach $\boldsymbol{V}=\overline{\boldsymbol{v}}+\boldsymbol{v}^{\prime}$ with mean velocity $\overline{\boldsymbol{v}}$ and mean value fluctuation $\boldsymbol{v}^{\prime}$. Furthermore, we neglect the effect of meridional circulations inside the star by assuming that the mean velocity field arises purely from stellar rotation (Lanza 2006). The equation for the angular velocity $\omega=v_{\phi} /(r \sin \theta)$ reads (see, e.g., Lanza 2005, 2006)

$$
\begin{aligned}
& \frac{\partial \omega}{\partial t}-\frac{1}{\rho r^{4}} \frac{\partial}{\partial r}\left(r^{4} \eta_{\mathrm{t}} \frac{\partial \omega}{\partial r}\right)-\frac{\eta_{\mathrm{t}}}{\rho r^{2}} \frac{1}{\left(1-\mu^{2}\right)} \frac{\partial}{\partial \mu}\left(\left(1-\mu^{2}\right)^{2} \frac{\partial \omega}{\partial \mu}\right) \\
& =S(r, \mu, t) .
\end{aligned}
$$

Here, $\eta_{\mathrm{t}}=\eta_{\mathrm{t}}(r)$ is the turbulent dynamical viscosity, $\mu=\cos \theta$. $S(r, \mu, t)$ is a source term that controls the temporal evolution of the angular momentum redistribution and is specified in Sect. 2.3.1. Equation (1) is solved assuming a stress-free boundary condition

$$
\left(\frac{\partial \omega}{\partial r}\right)_{r_{\mathrm{b}}, R}=0
$$

$r_{\mathrm{b}}$ denotes the base of the stellar convection zone, and $R$ is the radius of the star (Lanza 2006).

\subsection{Angular momentum equation}

\subsubsection{Model framework}

Introducing the state of rigid rotation $\Omega_{0}$, the angular velocity of the star can be written as

$\Omega(r, \mu, t)=\Omega_{0}+\omega(r, \mu, t)$, 
where $\omega(r, \mu, t)$ describes the deviation from rigid rotation (Lanza 2006). According to Lanza (2006), solutions of the angular velocity Eq. (1) have the form

$\omega(r, \mu, t)=\sum_{n=0}^{\infty} \alpha_{n}(t) \zeta_{n}(r) P_{n}^{(1,1)}(\mu)$,

where $P_{n}^{(1,1)}$ are Jacobian polynomials and the index $n$ is referred to as angular order. Furthermore, following Lanza (2006), the source term $S(r, \mu, t)$ can be developed into

$S(r, \mu, t)=\sum_{n=0}^{\infty} \beta_{n}(t) \zeta_{n}(r) P_{n}^{(1,1)}(\mu)$.

Based on the angular momentum conservation equation and using the properties of the Jacobian polynomials, Lanza (2006) derived a partial differential equation for the $\alpha_{n}, \beta_{n}$, and $\zeta_{n}$ functions that can be separated into two equations. They can be solved independently from each other, namely

$\frac{\mathrm{d} \alpha_{n}(t)}{\mathrm{d} t}-\beta_{n}(t)+\alpha_{n}(t) \lambda_{n}=0$,

which we refer to as temporal equation, as well as

$\frac{1}{\rho(r) r^{4}} \frac{\mathrm{d}}{\mathrm{d} r}\left(r^{4} \eta_{\mathrm{t}}(r) \zeta_{n}^{\prime}(r)\right)-n(n+3) \frac{\eta_{\mathrm{t}}(r)}{\rho(r) r^{2}} \zeta_{n}(r)+\lambda_{n} \zeta_{n}(r)=0$

which we refer to as the radial equation, and $\lambda_{n}$ are eigenvalues of Eq. (7). The radial Eq. (7) together with the boundary conditions (2) define a regular Sturm-Liouville problem in the interval $\left[r_{\mathrm{b}}, R\right]$ for $r_{\mathrm{b}}>0$ (Lanza 2006).

\subsubsection{Properties of the radial equation}

For any given $n$, an infinite number of eigenvalues exist. Therefore, Lanza (2006) introduced a new index $k$ (radial order) and denominated as $\lambda_{n k}$ the $k$ th eigenvalue of the $n$th radial equation. The first eigenvalue $\lambda_{00}$ is zero, the associated eigenfunction $\zeta_{00}$ vanishes, and all eigenvalues are positive (see Lanza 2006).

Accounting for the radial order $k$, Eq. (4) can be recast into

$\omega(r, \mu, t)=\sum_{k} \sum_{n=0}^{\infty} \alpha_{n k}(t) \zeta_{n k}(r) P_{n}^{(1,1)}(\mu)$,

and the radial Eq. (7) now reads

$\frac{1}{\rho r^{4}} \frac{\mathrm{d}}{\mathrm{d} r}\left(r^{4} \eta_{\mathrm{t}} \zeta_{n k}^{\prime}\right)-n(n+3) \frac{\eta_{\mathrm{t}}}{\rho r^{2}} \zeta_{n k}+\lambda_{n k} \zeta_{n k}=0$.

Imposing $|\omega| \ll \Omega_{0}$ (cf. Eq. (3)) and employing a linear approximation for the variation of the gravitational quadrupole moment (see Sect. 2.5), we only have to consider the cases $n=0$ and $n=2$ (Lanza 2006). Strictly speaking, in the case of a convective zone that spans the entire star $\left(r_{\mathrm{b}}=0\right)$, the regular SturmLiouville problem turns into a singular Sturm-Liouville problem. The boundary condition at the lower end of the domain must be replaced by a regularity condition, and a different treatment of Eq. (9) is required to deal with singularities at $r=0$. However, the stellar structure models we employ (see Sect. 3) all start at some radial coordinate $r>0$, which ensures $r_{\mathrm{b}}>0$ even for fully convective stars.

\subsubsection{Solving the radial equation}

The most essential input to solve the radial equations is a stellar structure model that provides the mass distribution within the star $M(r)$, the luminosity $L(r)$, the density $\rho(r)$, the temperature $T(r)$, and the mean molecular weight $m_{\mu}(r)$. Based on this input, we can derive the gravitational acceleration

$g(r)=\frac{G M(r)}{r^{2}}$

and the convective velocity employing mixing length theory $u_{\mathrm{c}}$

$u_{\mathrm{c}}(r)=\left(\frac{\alpha_{\mathrm{ml}} L(r)}{40 \pi r^{2} \rho(r)}\right)^{1 / 3}$

where we use a mixing length parameter $\alpha_{\mathrm{ml}}=1.5$ (Lanza 2005). The pressure scale height $h_{\mathrm{p}}$ is given by

$h_{\mathrm{p}}(r)=\frac{k_{\mathrm{B}} T(r)}{m_{\mathrm{H}} m_{\mu}(r) g(r)}$,

with Boltzmann's constant $k_{\mathrm{B}}$ and the hydrogen mass $m_{\mathrm{H}}$ (Lanza 2005). Finally, we can calculate the turbulent viscosity from

$\eta_{\mathrm{t}}(r)=\frac{1}{3} \rho(r) \alpha_{\mathrm{ml}} u_{\mathrm{c}}(r) h_{\mathrm{p}}(r)$

The stress-free boundary condition specified by Eq. (2) implies $\zeta_{n k}^{\prime}(r)=0$ at $r=r_{\mathrm{b}}$ and $r=R$ as boundary conditions for $\zeta_{n k}$. We assume that $\zeta_{n k}$ vanishes outside $\left[r_{\mathrm{b}}, R\right]$, that is, angular velocity variations only occur in the convection zone. Intermediate stellar structure values are calculated by a linear interpolation scheme. Solutions for $\zeta_{0 k}$ and $\zeta_{2 k}$ are computed with a shooting method (see, e.g., Fehlberg 1987; Press et al. 1992). While the boundary conditions imply $\zeta_{n k}^{\prime}\left(r_{\mathrm{b}}\right)=0$, the eigenfunction normalization at the bottom of the convective zone $\zeta_{n k}\left(r_{\mathrm{b}}\right)$ can be regarded as a free parameter of the model only restricted by $\zeta_{n k}\left(r_{\mathrm{b}}\right) \ll \Omega_{0}$. The effect of this parameter is addressed in Sect. 3.

Lanza (2006) adopted $\zeta_{n k}\left(r_{\mathrm{b}}\right)=0.01 \Omega_{0}$ throughout his calculations, that is to say, he normalized individual angular momentum redistribution modes to some level. In contrast, we solve the full temporal equation to find solutions for the $\alpha_{n k}(t)$ and $\beta_{n k}(t)$ functions, which allows us to calculate the evolution of the redistribution processes and consider superpositions of the elemental redistribution functions $\zeta_{n k}(r)$.

\subsection{Temporal equation}

\subsubsection{Framework}

Following Lanza (2006), solutions for the temporal Eq. (6) are

$\alpha_{n k}(t)=\exp \left(-\lambda_{n k} t\right) \int_{0}^{t} \beta_{n k}\left(t^{\prime}\right) \exp \left(\lambda_{n k} t^{\prime}\right) \mathrm{d} t^{\prime}+\alpha_{n k}(0)$,

where $\beta_{n k}$ is calculated from

$\beta_{n k}=E_{n k} \int_{r_{\mathrm{b}}}^{R} \int_{-1}^{1} \rho r^{4} S(r, \mu, t) \zeta_{n k} P_{n}^{(1,1)}\left(1-\mu^{2}\right) \mathrm{d} \mu \mathrm{d} r$. 
$E_{n k}$ is a normalization constant ${ }^{1}$ given by

$E_{n k}^{-1}=\int_{r_{\mathrm{b}}}^{R} \rho r^{4} \zeta_{n k}^{2} \mathrm{~d} r \int_{-1}^{1} P_{n}^{(1,1)} P_{n}^{(1,1)}\left(1-\mu^{2}\right) \mathrm{d} \mu$.

The integral involving the Jacobian polynomials simplifies to

$\int_{-1}^{1} P_{n}^{(1,1)} P_{n}^{(1,1)}\left(1-\mu^{2}\right) \mathrm{d} \mu=\frac{8(n+1)}{(2 n+3)(n+2)}$.

In a linear approximation for the quadrupole moment variation, we only have to consider the angular orders $n=0$ and $n=2$ (see Sect. 2.5) for which the term on the right-hand side can be evaluated as $4 / 3$ for $n=0$ and $6 / 7$ for $n=2$.

The source term $S$ in Eq. (1) describes angular momentum transfer by Reynolds stresses and magnetic torques and takes the form

$S(r, \mu, t)=-\frac{\operatorname{div} \tau}{\rho r^{2}\left(1-\mu^{2}\right)}$.

The vector $\tau_{i}$ has the components

$\tau_{i}=r \sin \theta\left(\Lambda_{i \phi}+\frac{1}{\tilde{\mu}}\left(B_{i} B_{\phi}+M_{i \phi}\right)\right)$.

$\Lambda_{i \phi}=\rho \overline{v_{i}^{\prime} v_{\phi}^{\prime}}$ is the Reynolds stress tensor, $\tilde{\mu}$ is the magnetic permeability, $B$ is the mean magnetic field, and $M_{i \phi}=\overline{B_{i}^{\prime} B_{\phi}^{\prime}}$ is the Maxwell stress tensor (Lanza 2005). Finally, we arrive at the expression

$\beta_{n k}=-E_{n k} \int_{-1}^{1} \int_{r_{b}}^{R} r^{2} \operatorname{div} \tau \zeta_{n k} P_{n}^{(1,1)} \mathrm{d} \mu \mathrm{d} r$

to calculate the $\beta_{n k}$ functions from which we can then calculate the $\alpha_{n k}$ functions necessary to describe the temporal evolution of the star's angular momentum distribution.

\subsubsection{Stellar parameter fluctuations}

As the Applegate mechanism is linked to and triggered by magnetic activity, we impose a phase factor of the form

$f(t)=\sin \left(\omega_{\text {act }} t\right)$

with $\omega_{\text {act }}=2 \pi / P_{\text {act }}$ to describe the fluctuations of all involved quantities where $P_{\text {act }}$ is the activity cycle period (see, e.g., Rüdiger et al. 2002). For the magnetic field, we assume a simple azimuthal structure with $B_{r}=0, B_{\theta}=0$ and

$B_{\phi}(t)=B_{\text {surf }} \sin \left(\omega_{\text {act }} t\right)$

accompanied by magnetic field fluctuations of the form

$B_{i}^{\prime}(r, t)=A_{\mathrm{B}} B_{\text {surf }} \sin \left(\omega_{\text {act }} t\right)$

1 The expression for the normalization constant can be derived by multiplying Eq. (5) with $\rho r^{4} \zeta_{n k} P_{n}^{(1,1)}\left(1-\mu^{2}\right)$, integrating as $\int_{r_{\mathrm{b}}}^{R} \int_{-1}^{1} \mathrm{~d} \mu \mathrm{d} r$ and making use of the orthogonality of $\zeta_{n k}$ for a fixed $n$ with respect to the weight function $\rho r^{4}$, and the orthogonality of the Jacobian polynomials. and velocity field fluctuations in the convection zone

$v_{i}^{\prime}(r, t)=A_{\mathrm{v}} u_{\mathrm{c}}(r) \sin \left(\omega_{\mathrm{act}} t\right)$,

where $A_{\mathrm{B}}$ and $A_{\mathrm{v}}$ are amplification coefficients and $B_{\text {surf }}$ is the surface magnetic field amplitude. Assuming that these equations describe proper long-term ensemble means and imposing that both $B_{i}^{\prime} B_{j}^{\prime}$ and $v_{i}^{\prime} v_{j}^{\prime}$ are independent statistics for $i \neq j$, we can make an ansatz for the Reynolds tensor

$\Lambda_{i \phi}(r, t)=\rho(r) A_{\mathrm{v}}^{2} u_{\mathrm{c}}^{2}(r) \sin ^{2}\left(\omega_{\mathrm{act}} t\right)$

as well as the Maxwell stress tensor:

$M_{i \phi}(r, t)=A_{\mathrm{B}}^{2} B_{\text {surf }}^{2} \sin ^{2}\left(\omega_{\text {act }} t\right)$.

The effect of a constant phase lag $\Delta \phi$ between the phase factor of different coordinates is addressed in Sect. 3.5. We note that the stellar parameter fluctuation description we adopt for our model does not come from dynamo theory or hydrodynamic calculation, but is rather a simplified ad hoc approach to generate cyclic Maxwell and Reynolds stresses.

\subsubsection{Solution of the temporal equation}

We can now evaluate the source term $S(r, \mu, t)$. For a vector field $\boldsymbol{F}(r, \theta, \phi)$ in spherical coordinates, the divergence takes the form

$\operatorname{div} \boldsymbol{F}=\left(\frac{1}{r^{2}} \frac{\partial}{\partial r}\left(r^{2} \boldsymbol{F}_{r}\right), \frac{1}{r \sin \theta} \frac{\partial}{\partial \theta}\left(\sin \theta \boldsymbol{F}_{\theta}\right), \frac{1}{r \sin \theta} \frac{\partial}{\partial \phi}\left(\boldsymbol{F}_{\phi}\right)\right)^{\mathrm{T}}$

For our given magnetic field configuration and fluctuation setup, the divergence of the vector $\tau$ can be calculated as

$\operatorname{div} \tau=\sqrt{1-\mu^{2}} \frac{1}{r^{2}} \frac{\partial}{\partial r}\left[r^{3} \tilde{B}_{r \phi}(r, t)\right]+2 \mu \tilde{B}_{\theta \phi}$,

where we defined

$\tilde{B}_{i \phi}(r, t)=\Lambda_{i \phi}(r, t)+\frac{B_{i}(r, t) B_{\phi}(r, t)}{\tilde{\mu}}+\frac{M_{i \phi}(r, t)}{\tilde{\mu}}$.

Using this, the function $\beta_{n k}(t)$ can be evaluated as

$\beta_{n k}=-E_{n k} \int_{r_{\mathrm{b}}}^{R} \zeta_{n k}(r) \frac{\partial}{\partial r}\left[r^{3} \tilde{B}_{r \phi}(r, t)\right] \mathrm{d} r \int_{-1}^{1} P_{n}^{(1,1)} \sqrt{1-\mu^{2}} \mathrm{~d} \mu$.

The second term from $\operatorname{div} \tau$ vanishes because of the symmetry of the $\mu$ integral. The remaining Jacobian polynomial integral can be evaluated analytically. First, we substitute $\mu=\sin u$ and use the identity $\sin ^{2} u+\cos ^{2} u=1$, which yields

$\int_{-1}^{1} P_{n}^{(1,1)} \sqrt{1-\mu^{2}} \mathrm{~d} \mu$
$=\int_{-\pi / 2}^{\pi / 2} P_{n}^{(1,1)}(\sin u) \mathrm{d} u-\int_{-\pi / 2}^{\pi / 2} P_{n}^{(1,1)}(\sin u) \sin ^{2}(u) \mathrm{d} u$.

Next, $P_{n}^{(1,1)}(\mu)$ can be written as

$P_{n}^{(1,1)}(\mu)=\frac{1}{n+2} \sum_{m=0}^{n} \frac{(n+m+2) !}{m !(n-m) !(m+1) ! 2^{m}}(\mu-1)^{m}$, 
Table 1. Results for the Jacobian integral as given by Eq. (31).

\begin{tabular}{cc}
\hline \hline Order $n$ & Result \\
\hline 0 & $\pi / 2$ \\
2 & $3 \pi / 32$ \\
4 & $5 \pi / 128$ \\
6 & $175 \pi / 8192$ \\
8 & $441 \pi / 32768$ \\
10 & $4851 \pi / 524288$ \\
\hline
\end{tabular}

and for $(\mu-1)^{m}$, we have

$(\mu-1)^{m}=\sum_{k=0}^{m}(-1)^{k} \frac{m !}{k !(m-k) !} \mu^{m-k}$.

Using

$\int_{-\pi / 2}^{\pi / 2} \sin ^{q}(u) \mathrm{d} u=\pi \frac{2^{-q-1} q !\left((-1)^{q}+1\right)}{(q / 2) !^{2}}$,

the integrals in Eq. (31) are given by

$$
\begin{aligned}
& \int_{-\pi / 2}^{\pi / 2} P_{n}^{(1,1)}(\sin u) \mathrm{d} u \\
& =\frac{\pi}{2(n+2)} \sum_{m=0}^{n} \frac{(n+m+2) !}{(n-m) !(m+1) ! 2^{2 m}} \sum_{k=0}^{m} \frac{(-2)^{k}}{k !} \frac{\left((-1)^{m-k}+1\right)}{((m-k) / 2) !^{2}}
\end{aligned}
$$

as well as

$$
\begin{aligned}
& \int_{-\pi / 2}^{\pi / 2} P_{n}^{(1,1)}(\sin u) \sin ^{2} u \mathrm{~d} u \\
& =\frac{\pi}{8(n+2)} \sum_{m=0}^{n} \frac{(n+m+2) !}{(n-m) !(m+1) ! 2^{2 m}} \\
& \quad \times \sum_{k=0}^{m} \frac{(-2)^{k}}{k !} \frac{\left((-1)^{m-k+2}+1\right)(m-k+1)(m-k+2)}{((m-k+2) / 2) !^{2}} .
\end{aligned}
$$

Because of the symmetry of the integrand, the integral vanishes for odd values of $n$, implying $\beta_{n k}=0$ and $\alpha_{n k}=0$. Using Eq. (35) and Eq. (36) allows us to explicitly calculate the Jacobian integral Eq. (31) (see Table 1). In order to normalize and calculate $\alpha_{n k}$, we have to make use of the initial conditions, which correspond to the state of rigid rotation. Formally, we have

$$
\Omega(r, 0,0)=\Omega_{0},
$$

which implies

$\omega(r, 0,0)=0$

and can be satisfied by demanding $\alpha_{n k}(0)=0$.

\subsection{Energy dissipation}

According to Lanza (2006), the variation of the rotational kinetic energy can be calculated from

$\Delta \mathcal{T}_{n k}=\frac{8 \pi(n+1)}{(2 n+3)(n+2)} \alpha_{n k}^{2} \int_{r_{\mathrm{b}}}^{R} \rho r^{4} \zeta_{n k}^{2} \mathrm{~d} r$,

with a kinetic energy dissipation rate $P_{\text {diss }}$ given by

$P_{\text {diss }}=-2 \sum_{k} \sum_{n} \lambda_{n k} \Delta \mathcal{T}_{n k}$

\subsection{Gravitational quadrupole moment variation}

According to Ulrich \& Hawkins (1981) and Lanza (2006), the variation of the quadrupole moment potential $\Delta \Phi_{12}$ can be calculated by solving

$$
\begin{aligned}
\frac{\partial^{2}\left(\Delta \Phi_{12}\right)}{\partial r^{2}}= & -\frac{2}{r} \frac{\partial\left(\Delta \Phi_{12}\right)}{\partial r}+\frac{6}{r^{2}}\left(\Delta \Phi_{12}\right)+\frac{4 \pi r^{2}}{M(r)} \\
& \times\left[\left(\frac{\mathrm{d} \rho}{\mathrm{d} r}\right)\left(\Delta \Phi_{12}\right)-\frac{\partial}{\partial r}\left(r^{2} \rho b_{2}\right)-r \rho a_{2}\right],
\end{aligned}
$$

where $M(r)$ is the integrated star mass up to a given radius $r$. The Lebovitz (1970) coefficients $a_{2}$ and $b_{2}$ are calculated from the radial eigenfunctions $\zeta_{n k}$

$a_{2}=\Omega_{0} \sum_{k} \frac{12}{7} \alpha_{2 k} \zeta_{2 k}-\frac{4}{3} \alpha_{0 k} \zeta_{0 k}$

and

$b_{2}=\Omega_{0} \sum_{k} \frac{2}{3} \alpha_{0 k} \zeta_{0 k}+\frac{4}{7} \alpha_{2 k} \zeta_{2 k}$.

Solutions for Eq. (41) must verify the condition

$\Delta \Phi_{12}^{\prime}+3 \Delta \Phi_{12} / R=0$

to match the outer gravitational potential (Lanza 2006).

In order to solve Eq. (41) with the boundary condition Eq. (44), we perform a shooting-method search and convert this boundary value problem into an initial value problem. The initial conditions are given by $\Delta \Phi_{12}(r)=C r^{2}$ and $\Delta \Phi_{12}^{\prime}(r)=2 C r$ for $r \rightarrow 0$. By performing subsequent integrations of the quadrupole moment Eq. (41) for varying trial constants $C$, we find a trial constant $C$ for which condition (44) holds.

From $\Delta \Phi_{12}(R)$, we can calculate the quadrupole moment variation $\Delta Q$ via (Lanza 2006)

$\Delta Q=-\frac{R^{3} \Delta \Phi_{12}(R)}{3 G}$,

giving a relative orbital period variation of Applegate (1992)

$\frac{\Delta P}{P}=-9 \frac{\Delta Q}{M a^{2}}$,

where $a$ is the semi-major axis of the binary and $M$ is the mass of the active component. 


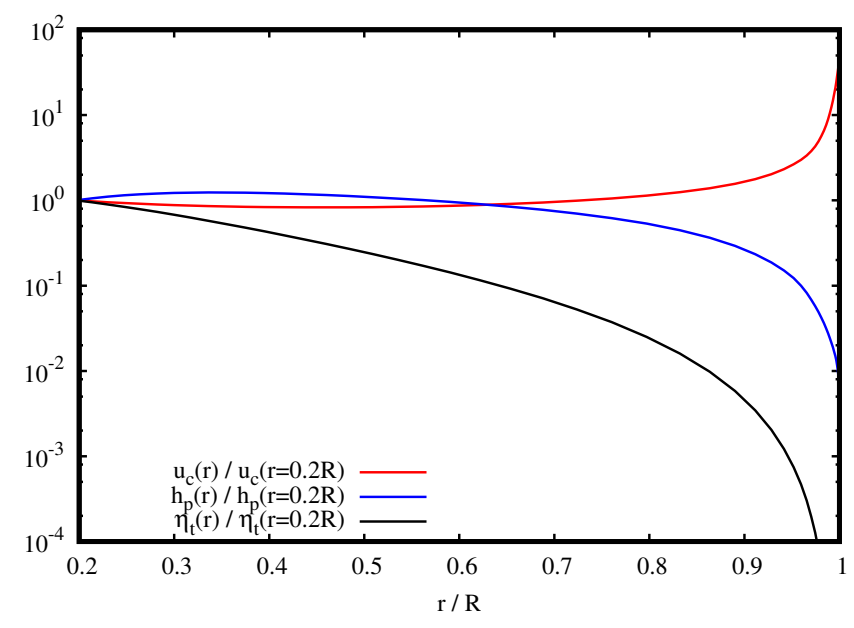

Fig. 1. Convective velocity profile $u_{\mathrm{c}}$, pressure scale height $h_{\mathrm{p}}$, and turbulent dynamical viscosity $\eta_{\mathrm{t}}$ calculated using an EZAMS model of HR 1099 and mixing length theory.

\section{Results}

We applied the formalism described in Sect. 2 to an EZAMS ${ }^{2}$ model of HR $1099^{3}\left(M=1.3 M_{\odot}\right.$, solar metallicity, time step $426, t=4.48 \mathrm{Gyr}, R \sim 4.05 R_{\odot}, L=8.62 L_{\odot}, \Omega_{0}=2.569$. $10^{-5} \mathrm{~s}^{-1}$, and depth of the convection zone $\left.r_{\mathrm{b}} / R=0.1968\right)$, which provides the necessary stellar data: radial mass profile $M(r)$, luminosity profile $L(r)$, density profile $\rho(r)$, temperature profile $T(r)$, and mean molecular weight $m_{\mu}(r)$, as well as the base of the convection zone $r_{\mathrm{b}}$. Using this, we can calculate derived quantities such as the radial gravitational acceleration profile $g(r)$, convective velocity $u_{\mathrm{c}}(r)$, pressure scale height $h_{\mathrm{p}}(r)$, and turbulent viscosity $\eta_{\mathrm{t}}(r)$, for which we employ mixing length theory with a mixing length parameter $\alpha_{\mathrm{ml}}=1.5$. We plot the radial profiles of $u_{\mathrm{c}}(r), h_{\mathrm{p}}(r)$ and $\eta_{\mathrm{t}}(r)$ in Fig. 1.

For the surface magnetic flux density, we assume $B_{\text {surf }}=$ $1 \mathrm{kG}$. Details on the calculation of the Maxwell stress tensor and Reynolds stress tensor are described in Sect. 2. We adopt $A_{v}=A_{B}=0.1$ as relative oscillation amplitudes for the velocity field and magnetic field inside the star.

\subsection{Analytical approach}

In order to understand the temporal evolution of the Applegate effect in the model presented here, we start with an analytical approach toward the most fundamental quantities involved in the mechanism. For a given set of functions that describe the oscillation of stellar parameters, the mechanism is controlled by the functions $\beta_{n k}$ and $\alpha_{n k}$, which can be calculated from the source term $S(r, \mu, t)$, which is in turn controlled by the function $\tilde{B}_{i \phi}(r, t)$ as given by Eq. (29). $\tilde{B}_{i \phi}(r, t)$ consists of three terms:

- a magnetic field term $B_{i} B_{\phi}(r, t)$ that controls the mean magnetic field,

- the Reynolds tensor $\Lambda_{i \phi}(r, t)$ that describes the velocity field fluctuations, and

- the Maxwell tensor $M_{i \phi}(r, t)$ that describes the magnetic field fluctuations.

Let $f(t)$ be a periodic function with period $P_{\text {act }}$ (implying $\left.f\left(t+P_{\text {act }}\right)=f(t)\right)$ that describes the temporal variation of the

\footnotetext{
2 http://www .astro.wisc.edu/ townsend/static.php?ref= ez-web

3 We chose HR 1099 for the sake of comparability with previous works by Lanza $(2005,2006)$ upon which our models is based.
}

stellar parameters involved in the Applegate mechanism, that is, $B_{i}^{\prime}(r, t)=B_{i}(r) f(t)$, and $v_{i}^{\prime}(r, t)=u_{c}(r) f(t)$. Furthermore, we employ a cyclic purely azimuthal magnetic field $B_{\phi}(t)=$ $B_{\text {surf }} f(t)$ with constant amplitude $B_{\text {surf }}$. We impose that all these quantities are tightly coupled and oscillate in phase. Under such conditions, all terms in $\widetilde{B}_{i \phi}(r, t)$ have the same time dependence, implying $\tilde{B}(r, t) \propto f(t)^{2}$ for fixed r. Substituting into Eq. (15) yields $\beta_{n k} \propto f(t)^{2}$. Given this result, we can evaluate Eq. (14): Following Lanza (2006) and employing that the first eigenvalues are typically associated with angular momentum redistribution modes on timescales shorter than the activity cycle of the star, we have $\alpha_{n k}=\beta_{n k} / \lambda_{n k}$, which yields $\alpha_{n k} \propto f(t)^{2}$.

Finally, we arrive at

$P_{\text {diss }} \propto f(t)^{4}$.

The main insight of this exercise is the change in periodicity: In case of sinusoidal variations with period $P_{\text {act }}$ in the magnetic field and the velocity field fluctuations, $\alpha_{n k}, \beta_{n k}$, and $P_{\text {diss }}$ oscillate with half of the activity period to the leading order ${ }^{4}$ which is the observed binary modulation period $P_{\bmod }$ as the quadrupole moment variation closely follows the dissipated energy (see following subsections). Another interesting result can be found by calculating the dimensionless quantity

$$
\frac{\tilde{\mu} \Lambda}{M}=\tilde{\mu} \frac{\int_{r_{\mathrm{b}}}^{R} A_{v}^{2} u_{c}^{2} \rho \mathrm{d} r}{\int_{r_{\mathrm{b}}}^{R} A_{B}^{2} B_{\text {surf }}^{2} \mathrm{~d} r},
$$

which compares the Reynolds tensor integral with the Maxwell tensor integral, quantifying the relative strength of the velocity field fluctuation amplitude versus the magnetic field fluctuation amplitude. Given $A_{v}=A_{B}=0.1$, a surface magnetic field of $B_{\text {surf }}=1 \mathrm{kG}$, and our EZAMS model of HR 1099, we have $\tilde{\mu} \Lambda / M \gg 1$, implying that the velocity fluctuations dominate the magnetic field fluctuations. The same holds for the zeroage main-sequence stars investigated in the parameter study in Sect. 3.6. As a result, the choice of $B_{\text {surf }}$ and $A_{B}$ does not significantly alter our results.

\subsection{Eigenvalues and radial eigenfunctions}

Figures 2 and 3 show the first four eigenfunctions $\zeta_{0 k}(r)$ and $\zeta_{2 k}(r)$ associated with the first four eigenvalues of $\lambda_{0 k}$ and $\lambda_{2 k}$ (see Table 2). A radial eigenfunction of order $k$ has $k$ nodes in $\left[r_{\mathrm{b}}, R\right]$ (see, e.g., Lanza 2006).

\subsection{Eigenfunction normalization and $k$ cutoff}

For a fixed $r_{\mathrm{b}}$ and assuming that all radial eigenfunction $\zeta_{n k}$ share one normalization parameter $\zeta_{n k}\left(r_{\mathrm{b}}\right)$, we find that the scaling relations $\omega \propto \zeta_{n k}\left(r_{\mathrm{b}}\right)$ and $P_{\text {diss }} \propto \zeta_{n k}\left(r_{\mathrm{b}}\right)^{2}$ hold. Consider $\zeta_{n k}(r)=\zeta_{n k}\left(r_{\mathrm{b}}\right) \xi_{n k}(r)$ with dimensionless functions $\xi_{n k}(r)$. Plugging into Eq. (9) and making use of the linearity of differentials yields

$\frac{1}{\rho r^{4}} \frac{\mathrm{d}}{\mathrm{d} r}\left(r^{4} \eta_{\mathrm{t}} \xi_{n k}^{\prime}\right)-n(n+3) \frac{\eta_{\mathrm{t}}}{\rho r^{2}} \xi_{n k}+\lambda_{n k} \xi_{n k}=0$,

4 Using the multiple-angle formula $\cos 4 x=1-8 \sin ^{2} x+8 \sin ^{4} x$, we see that $f(t)^{2}$ and $f(t)^{4}$ oscillate with the same amplitude and frequency, but are accompanied by a smaller oscillation with four times the frequency and $1 / 8$ the amplitude. 


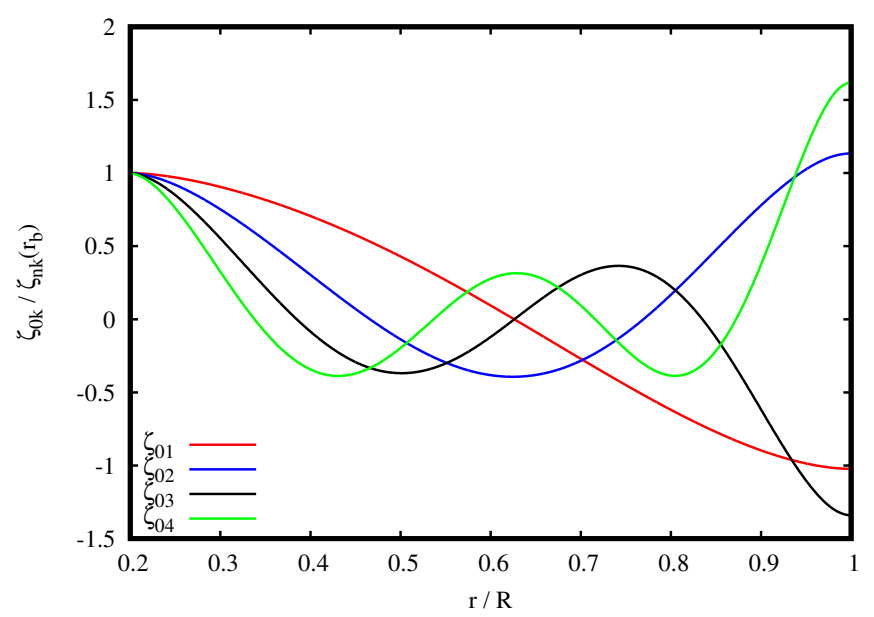

Fig. 2. Eigenfunctions $\zeta_{0 k}$ associated with the first four eigenvalues $\lambda_{0 k}$ calculated for HR 1099 (see Sect. 3.2). These functions represent elemental angular momentum redistribution modes.

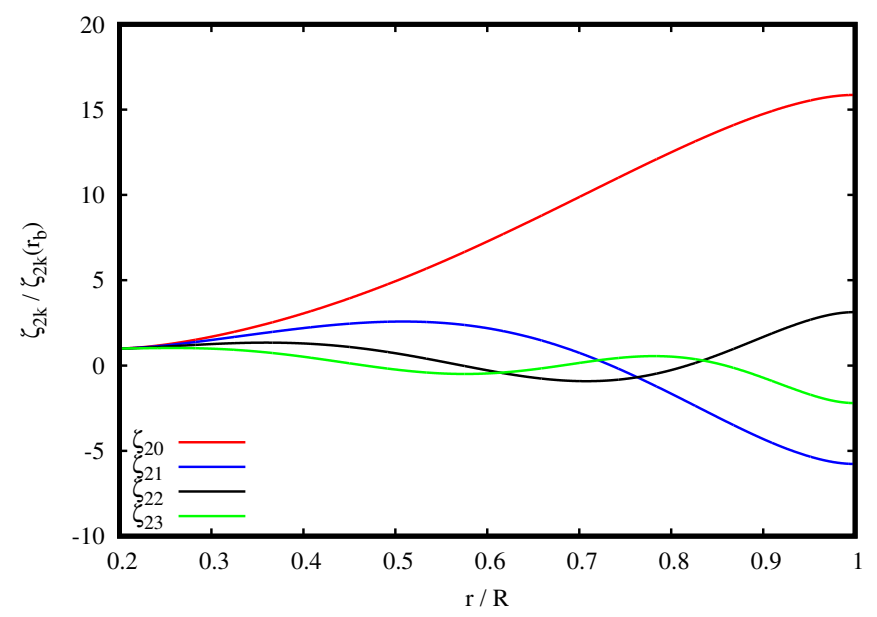

Fig. 3. Eigenfunctions $\zeta_{2 k}$ associated with the first four eigenvalues $\lambda_{2 k}$ calculated for HR 1099 (see Sect. 3.2). These functions represent elemental angular momentum redistribution modes.

which is equivalent to Eq. (9). This implies that for a fixed value of $r_{\mathrm{b}}, n$, and $k$, solutions for $\zeta_{n k}(r)$ with varying normalizations $\zeta_{n k}\left(r_{\mathrm{b}}\right)$ are multiples of one another. In particular, the eigenvalues $\lambda_{n k}$ are constant. With this, we obtain for the dissipated energy

$\Delta \mathcal{T}_{n k}=\zeta_{n k}^{2}\left(r_{\mathrm{b}}\right) \frac{8 \pi(n+1)}{(2 n+3)(n+2)} \alpha_{n k}^{2} \int_{r_{\mathrm{b}}}^{R} \rho r^{4} \xi_{n k}^{2} \mathrm{~d} r$,

and the kinetic energy dissipation rate becomes

$P_{\mathrm{diss}}=-2 \sum_{k} \sum_{n} \lambda_{n k} \Delta \mathcal{T}_{n k} \propto \zeta_{n k}^{2}\left(r_{\mathrm{b}}\right)$

as the eigenvalues $\lambda_{n k}$ are independent of the normalization chosen. In an analogous way, we can write

$\omega(r, \mu, t)=\zeta_{n k}\left(r_{\mathrm{b}}\right) \sum_{n=0}^{\infty} \sum_{k} \alpha_{n k}(t) \xi_{n k}(r) P_{n}^{(1,1)}(\mu)$

for the angular velocity variation inside the star, which indeed implies $\omega(r, \mu, t) \propto \zeta_{n k}\left(r_{\mathrm{b}}\right)$ because the functions $\alpha_{n k}$ are invariant under variations of $\zeta_{n k}\left(r_{\mathrm{b}}\right)$.
Table 2. Summary of the first ten eigenvalues of the radial equation for $n=0$ and $n=2$.

\begin{tabular}{ccc}
\hline \hline Order $k$ & $\lambda_{0 k} / s^{-1}$ & $\lambda_{2 k} / s^{-1}$ \\
\hline 0 & 0 & $3.54 \mathrm{E}-8$ \\
1 & $5.12 \mathrm{E}-8$ & $1.17 \mathrm{E}-7$ \\
2 & $1.39 \mathrm{E}-7$ & $2.27 \mathrm{E}-7$ \\
3 & $2.64 \mathrm{E}-7$ & $3.63 \mathrm{E}-7$ \\
4 & $4.28 \mathrm{E}-7$ & $5.27 \mathrm{E}-7$ \\
5 & $6.31 \mathrm{E}-7$ & $7.26 \mathrm{E}-7$ \\
6 & $8.73 \mathrm{E}-7$ & $9.64 \mathrm{E}-7$ \\
7 & $1.16 \mathrm{E}-6$ & $1.24 \mathrm{E}-6$ \\
8 & $1.48 \mathrm{E}-6$ & $1.56 \mathrm{E}-6$ \\
9 & $1.84 \mathrm{E}-6$ & $1.92 \mathrm{E}-6$ \\
10 & $2.24 \mathrm{E}-6$ & $2.32 \mathrm{E}-6$ \\
\hline
\end{tabular}

As we investigate the question whether the Applegate effect - if operative - can explain the observed period variations in close binaries, we choose the following normalization strategy: First, we start with $\zeta_{n k}\left(r_{\mathrm{b}}\right)=1$ and calculate the eigenvalues $\lambda_{n k}$ and the functions $\zeta_{n k}, \beta_{n k}$ and $\alpha_{n k}$ for $n=0$ and $n=2$ up to some maximum order $k_{\max }$. The maximum order is determined by the eigenvalues, which have the dimension of inverse time and are associated with angular momentum redistributions on a timescale of $1 / \lambda_{n k}$ (see Lanza 2005, 2006). For increasing orders of $n$ and $k$, these timescales gradually shrink until they fall below the typical travel time of a soundwave when crosses the convection zone, which we calculate from

$$
t_{\mathrm{c}}=\frac{\left(R-r_{\mathrm{b}}\right)^{2}}{\int_{r_{\mathrm{b}}}^{R} c_{\mathrm{s}} \mathrm{d} r},
$$

where the speed of sound is

$c_{\mathrm{s}}=\sqrt{5 P / 3 \rho}$

assuming monoatomic gas. From this point, we therefore assume that higher orders are not able to contribute to the global angular momentum redistribution process. Depending on the details of the stellar interior, typical $k$ cutoffs range between 50 for evolved subgiants and $>100$ for red dwarfs. Given a $\mathrm{k}$ cutoff and the eigenfunctions $\zeta_{n k}$ (starting with $\left.\zeta_{n k}\left(r_{\mathrm{b}}\right)=1\right), \beta_{n k}$ as well as $\alpha_{n k}$, we calculate the total energy dissipation $P_{\text {diss }}(t)$ and locate its maximum $P_{\text {diss,max }}$. Given this, we adjust the eigenfunction normalization factor $\zeta_{n k}\left(r_{\mathrm{b}}\right)$ in order to have a maximum dissipated power equal to $A_{\mathrm{P}} L_{\mathrm{HR} 1099}$. Using this normalization, the kinetic energy dissipation rate is limited to some fraction $A_{\mathrm{P}}$ of the stellar power output $L_{\mathrm{HR} 1099}$, which is typically on the order of $10 \%$, and we can finally calculate the corresponding angular velocity variations inside the star.

\subsection{Angular velocity variation and energy dissipation}

The dissipated energy is calculated from Eq. (51) up to some radial order $k$. For $k=48$, the timescale associated with the respective angular momentum redistribution mode is shorter than the calculated soundwave travel time. We fix the kinetic energy fluctuation limit to $A_{\mathrm{P}}=0.1$ as described in the previous subsection. When $\alpha_{n k}$ and $\zeta_{n k}$ are determined and our normalization to limit the kinetic energy dissipation is applied, we can calculate the effective angular velocity variation inside the star as functions of both time and stellar radius. In Fig. 4 we present the 


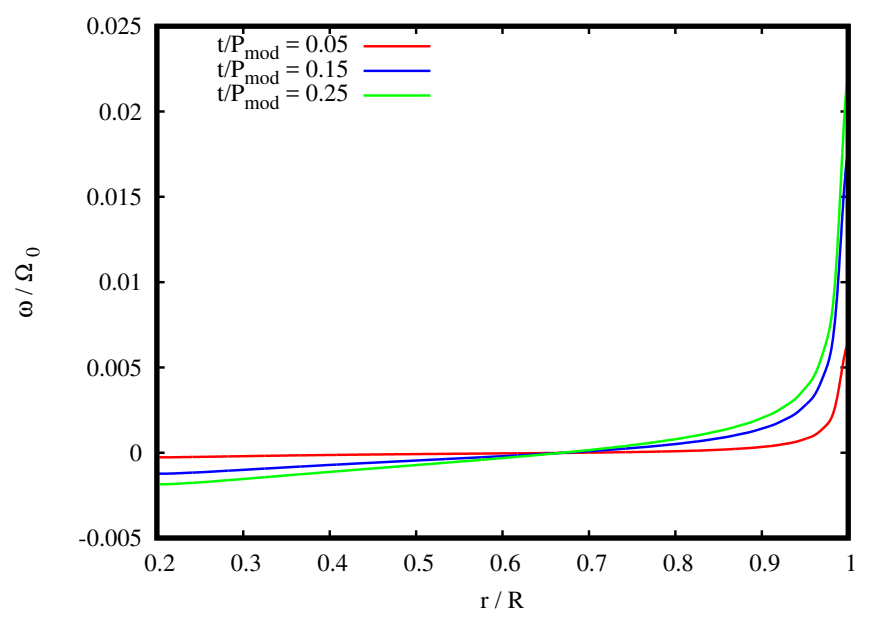

Fig. 4. Evolution of the equatorial angular velocity variation inside HR 1099 computed for three different times.

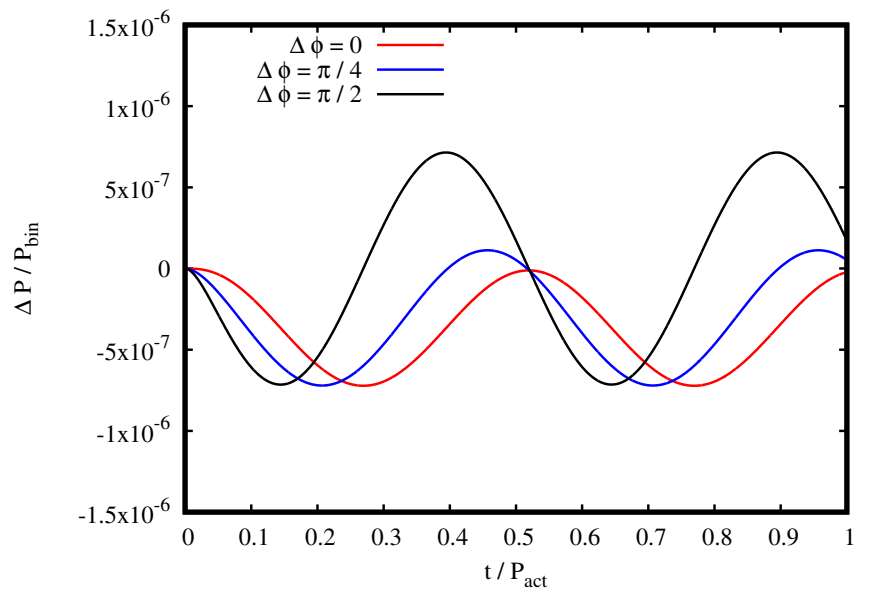

Fig. 5. Binary period variation amplitude calculated for a surface magnetic field of $1 \mathrm{kG}$, a cycle length of $P_{\text {act }}=2 P_{\text {mod }}$, a magnetic field fluctuation amplitude of $A_{B}=0.1$, and a velocity field fluctuation amplitude $A_{V}=0.1$ for three different cases of phase shift $\Delta \phi$ between the radial and azimuthal fluctuations.

angular velocity changes inside the star for three different times between $t=0.05 P_{\text {mod }}$ and $t=0.25 P_{\text {mod }}$. Starting from the state of rigid rotation at $t=0$, the angular velocity variation builds up continuously with peak fluctuations of a few percent in the outermost layers of the star.

\subsection{Period modulation}

In Fig. 5 we plot the calculated period variation in HR 1099 for $B_{\text {surf }}=1 \mathrm{kG}$, a cycle length of $P_{\text {act }}=2 P_{\text {mod }}$, a magnetic field fluctuation amplitude of $A_{B}=0.1$, and a velocity field fluctuation amplitude $A_{V}=0.1$ for three different cases of phase lag $\Delta \phi$ between the radial and the azimuthal velocity and magnetic field fluctuations. The dissipated energy is limited to $A_{P}=0.1$ of the star's luminosity. For $\Delta \phi=0$, the oscillation is purely negative, that is, the quadrupole moment changes have a positive sign. On the other hand, for $\Delta \phi=\pi / 2$, the period modulation oscillates between the positive and the negative regime, which is indicative of a cyclic transition between an oblate and a prolate state. Figure 6 shows the expected level of period variation in case of a surface magnetic field of $1 \mathrm{kG}$ and varying fluctuation parameters $A_{V}, A_{B}$, and $A_{P}$

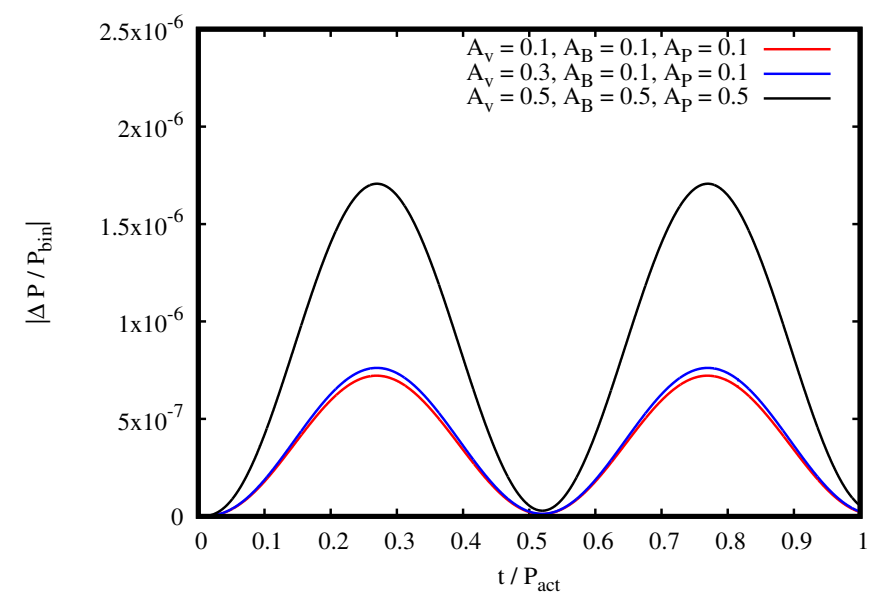

Fig. 6. Binary period variation in HR 1099 calculated for a surface magnetic field of $1 \mathrm{kG}$, a cycle length of $P_{\text {act }}=2 P_{\bmod }$, no phase shift, and varying fluctuation parameters $A_{V}, A_{B}$, and $A_{P}$. In a conservative scenario with $A_{P}=A_{V}=A_{B}=0.1$, the calculated binary period modulation amplitude is two orders of magnitude below the observed values $\left(\sim 10^{-4}\right)$. Increasing the velocity field fluctuation amplitude to $A_{V}=0.3$ at constant power dissipation $A_{P}=0.1$ only results in a slight increase of $\triangle P / P$. Even for the rather extreme case of $A_{P}=A_{V}=$ $A_{B}=0.5$, the produced period variations disagree with observations (see Frasca \& Lanza 2005).

(see Sect. 2.2.1). Even with fluctuation amplitudes as high as $A_{V}=A_{B}=A_{P}=0.5$, which are not observed in HR 1099 (see Frasca \& Lanza 2005), the resulting binary period variation amplitudes are still off by two orders of magnitude; no physically reasonable parameter range yields period variations as high as those observed in the system $\left(9.0 \times 10^{-5}\right.$, Frasca \& Lanza 2005). Given that the redistribution processes are almost entirely governed by convection (see Sect. 3.1), the only degree of freedom left to produce larger period variations is the convective velocity inside the star, for instance, through the mixing-length parameter $\alpha_{\mathrm{ml}}$. Reproduction of the observed modulation period of $35 \mathrm{yr}$ imposes an activity cycle length of twice that value as the model predicts a 2:1 relation between the observed binary modulation period and the activity cycle length. Therefore, if the observed period variations are energetically and mechanically feasible, we would expect an activity cycle in HR 1099 of roughly $70 \mathrm{yr}$, while several authors claim to have found cycle lengths between $14.1 \pm 0.3 \mathrm{yr}$ and $19.5 \pm 2 \mathrm{yr}$ (see Lanza et al. 2006; Muneer et al. 2010; Perdelwitz et al. 2018, and references therein).

\subsection{Active component mass parameter study}

We showed in the previous sections that RS CVns such as HR 1099 are not expected to produce significant levels of period modulation. We now expand our analysis to precataclysmic PCEB systems (see, e.g., Zorotovic \& Schreiber 2013), which have been proposed as potential Applegate systems by Völschow et al. (2016).

Consider a tidally locked binary system with $a=1 R_{\odot}$, consisting of a typical white dwarf primary with $M_{\mathrm{WD}}=0.5 M_{\odot}$ and a zero-age main-sequence secondary with varying mass. Just as in the previous sections, we assume a constant magnetic field of $B_{\text {surf }}=1 \mathrm{kG}$ and fix the fluctuation parameters to $A_{P}=A_{V}=$ $A_{B}=0.1$ with a phase lag $\Delta \phi=0$. For the modulation period, we assume $P_{\text {mod }}=15 \mathrm{yr}$. We varied the mass of the active component between $0.1 M_{\odot}$ and $0.6 M_{\odot}$ and calculated the amplitude 


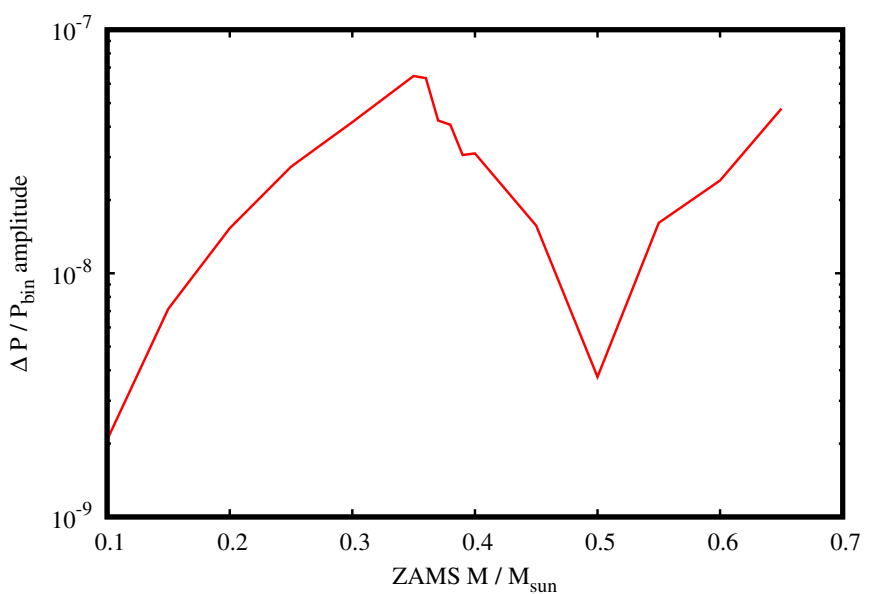

Fig. 7. Amplitude of binary period variation calculated for a surface magnetic field of $1 \mathrm{kG}$, cycle length of $P_{\text {act }}=2 P_{\text {mod }}$, magnetic field fluctuation amplitude of $A_{B}=0.1$, velocity field fluctuations of $A_{V}=$ 0.1 , and varying active component mass. The noticeable decrease in period modulation at $0.37 M_{\odot}$ coincides with the transition from fully convective to radiative-core stars.

of the orbital period variation using an EZAMS model of age $t=0$. Figure 7 plots the expected period variation amplitude as a function of the active component mass. Between $0.1 M_{\odot}$ and $0.36 M_{\odot}$, we see a clear increasing trend toward higher binary period variation levels. For fluctuation parameters slightly above the fiducial $10 \%$ level, stars between $0.30 M_{\odot}$ and $0.36 M_{\odot}$ are expected to produce amplitudes $\simeq 10^{-7}$, which is a typical order of magnitude observed in PCEB systems (Parsons et al. 2010; Zorotovic \& Schreiber 2013; Völschow et al. 2016), while stars outside of this range only support low levels of period variations $\lesssim 10^{-7}$. For all simulated systems, the peak angular velocity variations are $\lesssim 1 \%$.

The physical explanation for the peak around $M \simeq 0.35 M_{\odot}$ is the emergence of a radiative core, which shifts the boundary of the convective zone outward and reduces the total mass and angular momentum involved in the redistribution process. In Fig. 8 we plot the bottom of the convective zone as function of mass. Stars with $M \gtrsim 0.37 M_{\odot}$ develop a radiative core, which reduces the domain available for angular momentum redistribution processes. While the calculated binary period modulation amplitudes again increase toward higher masses, the upper end of the investigated mass range may be affected by Roche-lobe overflow, which introduces additional effects that are not considered in our model. We also recall that fully convective stars with $r_{\mathrm{b}} / R=0$ are strictly speaking not covered by the regular Sturm-Liouville problem, but the structure files adopted in our calculations for stars up to $M=0.36 M_{\odot}$ have relative convection zone depths of $r_{\mathrm{b}} / R \simeq 0.01$.

\section{Conclusions and discussion}

We presented a model that connects periodic fluctuations in the magnetic field and velocity field of the convection zone to periodic modulations of the quadrupole moment of the star, resulting in cyclic modulations of the binary period. Using the example of HR 1099, which has been discussed as one of the most promising Applegate candidates, we calculated the angular momentum redistribution modes $\zeta_{n k}(r)$ and considered the angular equation to calculate the $\alpha_{n k}(t)$ and $\beta_{n k}(t)$ functions that are required to calculate the temporal evolution of the dissipated energy and the quadrupole moment, assuming that the

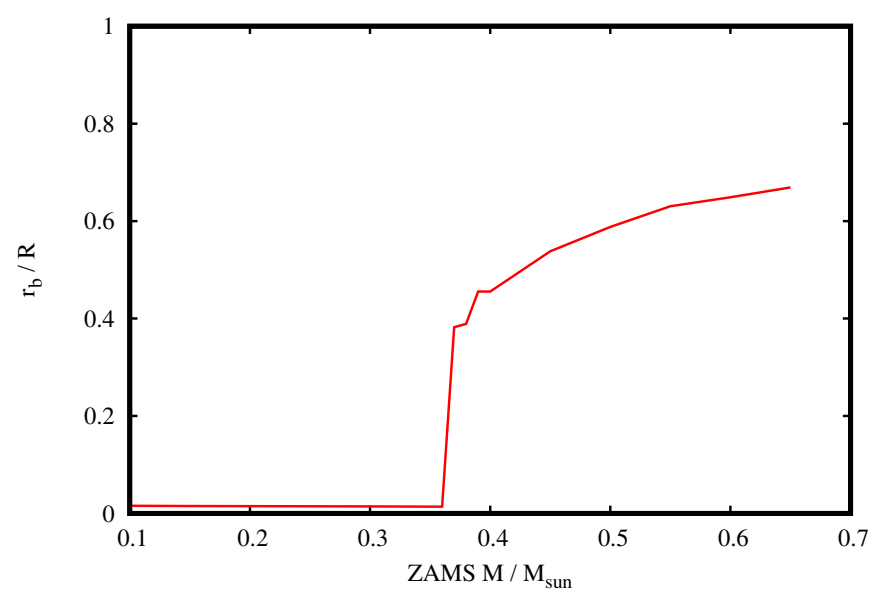

Fig. 8. Bottom of the convection zone $r_{\mathrm{b}}$ as function of mass in our EZAMS stellar structure models. Stars up to $0.36 M_{\odot}$ are fully convective with non-zero convection velocities down to the innermost grid points. For higher masses, EZAMS predicts the emergence of a radiative core that takes up increasing fractions of the central region and reduces the total mass participating in the angular momentum redistribution processes.

star dedicates a fiducial fraction of $A_{P}=0.1$ of its luminosity to drive the Applegate mechanism. The order up to which we calculate the eigenfunctions is determined by a simple soundwave travel time argument. Our constant azimuthal field ansatz leads to a simple source function only controlled by the $\Lambda_{r \phi}$ and $M_{r \phi}$ components of the Reynolds and Maxwell tensors, where the former clearly dominates in the case of simple harmonic velocity and magnetic field fluctuations, which we control through the $A_{B}$ and $A_{V}$ parameters with fiducial values of 0.1 .

In this model, harmonic oscillations of the magnetic field fluctuations and velocity field fluctuations on a timescale of the activity cycle $P_{\text {act }}$ result in orbital period variations on a timescale of $P_{\text {mod }}=0.5 P_{\text {act }}$. In other words, we expect that the activity cycle of any Applegate candidate satisfying our assumptions is double the observed binary modulation period, whereas alternative models predict a different ration (see, e.g., Applegate 1992; Lanza et al. 1998; Lanza \& Rodonò 2004).

Furthermore, depending on the phase shift between the radial fluctuations and the azimuthal fluctuations, purely negative, positive or mixed quadrupole moment changes may be observed similar to findings by Rüdiger et al. (2002).

In line with previous work, we confirm that HR 1099 is not expected to produce period variations on the level observed today through the Applegate mechanism, which would require unphysically high field fluctuation amplitudes, angular velocity changes, and dissipated power. This is consistent with previous findings by Lanza $(2005,2006)$, who did not explicitly consider the temporal evolution of the mechanism.

While HR 1099 in particular and RS CVn systems in general seem highly unlikely Applegate candidates, our extensive parameter study identified short-period PCEBs with active component masses between $0.30 M_{\odot}$ and $0.36 M_{\odot}$ as the strongest Applegate candidates with expected period variation amplitudes similar to those typically observed in such systems, which are between $10^{-7}$ and a few $10^{-6}$ (see, e.g., Zorotovic \& Schreiber 2013; Völschow et al. 2016). Consequently, we propose a careful reanalysis of PCEB systems with cyclic period variations to exclude the planetary hypothesis and find direct observational evidence that intrinsic effects such as quadrupole moment changes are at work. 
To distinguish between Applegate modulations and period variations caused by planets through the light travel time effect (see, e.g., Pribulla et al. 2012), long-term measurements are required to determine the correlations between activity indicators, luminosity variations that are due to photospheric temperature changes, and the binary period modulation (Applegate 1992, ). Changes in the rotational profile on the $1 \%$ level may lead to tiny deformations on a similar order of magnitude as the active component oscillates between an oblate and a prolate state, which is also expected to correlate with the activity cycle (Applegate 1992; Lanza et al. 1998; Rüdiger et al. 2002 ,). However, matters are complicated by spots and tidal deformations.

The model presented here is a significant step forward to understand the physics of the Applegate scenario. Nevertheless, it is still based on simplifying assumptions, such as a purely azimuthal magnetic field and the adoption of a mean-field framework. The Reynolds and Maxwell stress tensors do not selfconsistently follow from a dynamo model either, but we adopt a (physically motivated) prescription for the kinetic and magnetic fluctuations that is externally imposed. Similarly, this approach still neglects effects such as viscosity quenching as a result of fast rotation, which may reduce the dissipated power and allow for higher levels of period variation in rapidly rotating systems (see Lanza 2006). Furthermore, our model does not guarantee the hydrodynamical stability of the differential rotation profile that emerges during the oscillation, although the maximum relative deviations from the state of rigid rotation are on the $2 \%$ level in HR 1099 and well below the 1\% level in our PCEB parameter study (see Knobloch \& Spruit 1982). Another limitation ist that we do not account for meridional circulation or for the effect of energy advection inside the star. Finally, additional quadrupole moment changes may occur as results of anisotropic Lorentz forces inside the star (Lanza et al. 1998).

While we cannot draw a final conclusion at this point, our framework nevertheless provides the most complete assessment of the Applegate mechanism so far, and it shows that a consideration of physical processes allows determining under which conditions the Applegate mechanism may be feasible. Our model yields testable predictions, suggesting that the Applegate mechanism may be favored for certain PCEB systems and disfavored in HR 1099. These predictions should be tested both on the observational side and through further refinement of the model to provide a clear picture where the Applegate mechanism is able to work.
Acknowledgements. We would like to express our gratitude to the anonymous referee for carefully reading our manuscript and providing us with valuable advice that improved the quality of our work. DRGS thanks for funding via Fondecyt regular (project code 1161247), the "Concurso Proyectos Internacionales de Investigación, Convocatoria 2015" (project code PII20150171) and the BASAL Centro de Astrofísica y Tecnologías Afines (CATA) PFB-06/2007.

\section{References}

Applegate, J. H. 1992, ApJ, 385, 621

Applegate, J. H., \& Patterson, J. 1987, ApJ, 322, L99

Beuermann, K., Dreizler, S., \& Hessman, F. V. 2013, A\&A, 555, A133

Brinkworth, C. S., Marsh, T. R., Dhillon, V. S., \& Knigge, C. 2006, MNRAS, 365,287

Faulkner, J. 1971, ApJ, 170, L99

Fehlberg, E. 1987, ZAMM - J. Appl. Math. Mech. / Z. Angew. Math. Mech., 67, 367

Frasca, A., \& Lanza, A. F. 2005, A\&A, 429, 309

Gray, D. F., \& Baliunas, S. L. 1994, ApJ, 427, 1042

Hall, D. S. 1991, ApJ, 380, L85

Han, Z.-T., Qian, S.-B., Irina, V., \& Zhu, L.-Y. 2017, AJ, 153, 238

Hardy, A., Schreiber, M. R., Parsons, S. G., et al. 2015, ApJ, 800, L24

Horner, J., Wittenmyer, R. A., Hinse, T. C., et al. 2013, MNRAS, 435, 2033

Knobloch, E., \& Spruit, H. C. 1982, A\&A, 113, 261

Kraft, R. P., Mathews, J., \& Greenstein, J. L. 1962, ApJ, 136, 312

Lanza, A. F. 2005, MNRAS, 364, 238

Lanza, A. F. 2006, MNRAS, 369, 1773

Lanza, A. F., \& Rodonò, M. 1999, A\&A, 349, 887

Lanza, A. F., \& Rodonò, M. 2004, Astron. Nachr., 325, 393

Lanza, A. F., Rodono, M., \& Rosner, R. 1998, MNRAS, 296, 893

Lanza, A. F., Piluso, N., Rodonò, M., Messina, S., \& Cutispoto, G. 2006, A\&A, 455,595

Lebovitz, N. R. 1970, ApJ, 160, 701

Matese, J. J., \& Whitmire, D. P. 1983, A\&A, 117, L7

Muneer, S., Jayakumar, K., Rosario, M. J., Raveendran, A. V., \& Mekkaden, M. V. 2010, A\&A, 521, A36

Nasiroglu, I., Goździewski, K., Słowikowska, A., et al. 2017, AJ, 153, 137

Navarrete, F. H., Schleicher, D. R. G., Fuentealba, J. Z., \& Völschow, M. 2018, A\&A, 615, A81

Parsons, S. G., Marsh, T. R., Copperwheat, C. M., et al. 2010, MNRAS, 407, 2362

Parsons, S. G., Gänsicke, B. T., Marsh, T. R., et al. 2013, MNRAS, 429, 256

Perdelwitz, V., Navarrete, F.H., Zamponi, J., et al. 2018, A\&A, 616, A161

Press, W. H., Teukolsky, S. A., Vetterling, W. T., \& Flannery, B. P. 1992, Numerical recipes in FORTRAN. The art of scientific computing, 963

Pribulla, T., Vaňko, M., Ammler-von Eiff, M., et al. 2012, Astron. Nachr., 333, 754

Qian, S.-B., Liu, L., Liao, W.-P., et al. 2011, MNRAS, 414, L16

Rüdiger, G., Elstner, D., Lanza, A. F., \& Granzer, T. 2002, A\&A, 392, 605

Ulrich, R. K., \& Hawkins, G. W. 1981, ApJ, 246, 985

Verbunt, F., \& Zwaan, C. 1981, A\&A, 100, L7

Völschow, M., Schleicher, D. R. G., Perdelwitz, V., \& Banerjee, R. 2016, A\&A, 587, A34

Zorotovic, M., \& Schreiber, M. R. 2013, A\&A, 549, A95 